\title{
Erratum to: Computationally tractable approximate and smoothed Polya trees
}

\author{
William Cipolli III ${ }^{1}$. Timothy Hanson ${ }^{1}$
}

Published online: 18 October 2016

(C) Springer Science+Business Media New York 2016

\section{Erratum to: Stat Comput}

\section{DOI 10.1007/s11222-016-9652-3}

The publisher regrets errors on equation breaks in the original publication. The correct equations are as shown below:

Section 2.2, after 10th paragraph,

$c^{*} \mid \mathcal{Y} \sim \Gamma\left(a_{c}+\frac{2^{J}-2}{2}, b_{c}+\frac{1}{4} \sum_{j=2}^{2^{J}} j^{2} \sum_{k=1}^{2^{j}-1}\left(\log \frac{Y_{j, 2 k-1}}{1-Y_{j, 2 k-1}}\right)^{2}\right)$.

Section 2.4, after 2nd paragraph

$\alpha^{-2} \mid \mathbf{q}, \mu, \sigma \sim \Gamma\left(a+\frac{n}{2}, a \sigma^{2}+\frac{1}{2} \sum_{i=1}^{n} \frac{\left(y_{i}-\mu-\sigma t_{q_{i}}\right)^{2}}{d_{q_{i}}^{2}}\right)$.
Section 2.5, after 2nd paragraph,

$\alpha^{-2} \mid \mathbf{q}, \gamma, \sigma, \mathbf{y} \sim \Gamma\left(a+\frac{n}{2}, a \sigma^{2}+\frac{1}{2} \sum_{i=1}^{n} \frac{\left(y_{i}-\mathbf{z}_{i}^{\prime} \gamma-\sigma t_{q_{i}}\right)^{2}}{d_{q_{i}}^{2}}\right)$.

Section 2.5, after 3rd paragraph,

$S_{\mathbf{z}}(t \mid \mu, \sigma, \mathcal{Y}, \alpha)=1-\sum_{k=1}^{2^{J}} p_{\mathcal{Y}}(k) \Phi\left(\log t \mid \mathbf{z}^{\prime} \gamma+\sigma t_{k}, \alpha^{2} d_{k}^{2}\right)$

The online version of the original article can be found under doi:10.1007/s11222-016-9652-3.

William Cipolli III

william.cipolli@gmail.com

1 Department of Statistics, University of South Carolina,

Columbia, SC, USA 